\title{
COVID-19 related complete blood count changes among asymptomatic pregnant women
}

\author{
Waleed F. Gharib, ${ }^{1}$ Hesham Nasser, ${ }^{2,3}$ Ahmed M. A. Sobh, ${ }^{4}$ Rasha E. Khamees ${ }^{1}$
}

Keywords: COVID-19; pregnancy; blood count

\begin{abstract}
Objective: To evaluate complete blood count (CBC) changes that suggest coronavirus disease-2019 (COVID-19) among asymptomatic pregnant women attending routine antenatal care

Methods: A cross-sectional study included 187 healthy pregnant women who were attending the antenatal care clinic of a tertiary University hospital between March and June 2020. After a thorough history and examinations, a venous blood sample was taken from each participant for complete and differential blood counts. Those who showed CBC findings suggestive of COVID-19 were further scheduled for a nasopharyngeal swab for detection of SARSCoV-2 specific antigens through polymerase chain reaction (PCR).
\end{abstract}

Results: We found 5.3\% ( $n=10)$ of the study population showed $C B C$ changes that are suggestive of COVID-19. When they were scheduled for nasopharyngeal swab for a PCR confirmatory test, $30 \%(n=3)$ of them were PCR positive (which represented $1.6 \%$ of the entire study population). The most frequently encountered COVID-19-suggestive change in peripheral blood leukocyte differential counts was leucopenia (100\%), followed by decreased eosinophil count (50\%), then neutropenia and lymphocytopenia (30\%).

Conclusions: Certain differential leucocyte count changes (leucopenia, neutropenia, lymphocytopenia and decreased eosinophil count) among asymptomatic pregnant women might be related to COVID-19 infection and may indicate a need for further testing.

${ }^{1}$ Department of Obstetrics \& Gynecology, Faculty of Medicine, Suez-Canal University, Ismailia, Egypt.

${ }^{2}$ Department of Clinical Pathology, Faculty of Medicine, Suez-Canal University, Ismailia, Egypt.

${ }^{3}$ Research Center for Human Retrovirus Infection, Kumamoto University, Japan

${ }^{4}$ Department of Obstetrics \& Gynecology, Faculty of Medicine, Assiut University, Assiut, Egypt.

Please cite this paper as: Gharib WF, Nasser H, Sobh AMA, Rasha E. Khamees RE. COVID-19 related complete blood count changes among asymptomatic pregnant women. Proc Obstet Gynecol. 2021;10(2):Article 5 [ 9 p.]. Available from: http://ir.uiowa.edu/ Free full text article.

Corresponding author: Dr. Ahmed M. A. Sobh, MD, Professor at Department of Obstetrics and Gynecology, Assiut University, Egypt, Women Health Hospital, 71111, Assiut Egypt. Cellular: +20 1553880089, Tel: +20 882414631 , Fax: +20 88 2419211. E-mail: ahmedsobh485@gmail.com

Financial Disclosure: The authors report no conflict of interest.

Copyright: (c) 2021 Gharib et al. This is an open-access article distributed under the terms of the Creative Commons Attribution License, which permits unrestricted use, distribution, and reproduction in any medium, provided the original author and source are credited. 
Proceedings in Obstetrics and Gynecology, 2021;10(2):5

\section{Introduction}

Coronaviruses are a family of pathogens that can potentially infect both humans and animals. They produce several clinical entities such as the common cold, severe acute respiratory syndrome (SARS) and Middle East respiratory syndrome (MERS). ${ }^{1}$ The first known human-infecting coronaviruses were called 229E and OC43. They generally cause very mild infections. However, after more severe outbreaks of SARS and MERS, this virus family has received markedly increased attention and has been subject to extensive study. ${ }^{2}$

A novel coronavirus was identified by the end of 2019 as the cause of several forms of pneumonia in Wuhan, Hubei Province of China, resulting in an epidemic throughout China. The pathogen was identified as severe acute respiratory syndrome coronavirus 2 (SARS-CoV-2), and the disease was identified as coronavirus disease-2019 (COVID-19). In March 2020, with the rapid worldwide spread across numerous countries, the COVID-19 outbreak was declared a pandemic by the World Health Organization (WHO). ${ }^{3}$

SARS-CoV-2 is an enveloped, positivestranded RNA virus with nucleocapsid. It is a beta coronavirus in the same subgenus as the severe acute respiratory syndrome (SARS) virus (as well as several bat coronaviruses). The receptor-binding gene region is very similar to that of the SARS coronavirus, and they were both found to use the same receptor for cell entry, the angiotensin-converting enzyme 2 (ACE2) receptor. ${ }^{4}$

COVID-19 presents with heterogeneous clinical features that markedly vary in severity. About $81 \%$ of cases present with mild disease. However, up to $14 \%$ of cases are moderate to severe and present with dyspnea, respiratory frequency $\geq 30 / \mathrm{min}$, blood oxygen saturation $(\mathrm{SpO} 2) \quad \leq 93 \%$, and percentage of oxygen supplied (fraction of inspired oxygen, $\mathrm{FiO} 2$ ) $<300$, and/or lung infiltrates $>50 \%$ within 24 to 48 hours. Critical infections represent only $5 \%$ of cases and include presentation as septic shock, respiratory failure and/or multiple organ failure. ${ }^{5}$

The rate of infection with SARS-CoV-2 in the study region at the time the study was conducted was underestimated. However, official reports claimed that it occurred in less than $1 \%$ of the population. Therefore, the current study aims to evaluate the complete blood count (CBC) changes that suggest COVID-19 infection among asymptomatic pregnant women attending routine antenatal care

\section{Materials and Methods}

This cross-sectional study was conducted at the antenatal care clinic of a tertiary University hospital between March and June 2020. All participants gave informed written consent before inclusion in the study. The study included 187 healthy pregnant females between the age of 18 and 40 years, irrespective of gestational age, who presented for routine antenatal care. 
After informed consent had been obtained, all the study participants underwent detailed history taking, and general, chest, abdominal and local examinations. The aim of the detailed history and examinations was to assure the absence of any obstetric complications or chronic medical conditions. An emphasis was made during history taking and examinations to exclude any participant who were symptomatic for COVID-19 infection. The presence of any of the following: fever, dry cough, tiredness, muscle aches and pains, sore throat, diarrhea, conjunctivitis, headache, loss of taste or smell, skin rash, discoloration of fingers or toes, difficulty in breathing or shortness of breath, chest pain or deteriorated general condition, in the previous 14 days preceding enrollment in the study was taken as suggestive of COVID-19, and the participant was excluded from the study.

After assuring proper inclusion of participants as previously detailed, a venous blood sample ( $3 \mathrm{~mL}$, to $\mathrm{K}^{+}$EDTA containing tube) was taken from each of the participants for complete and differential blood counts, using an automated cell counter as well as stained peripheral blood smear microscopy, with emphasis on differential leucocyte count. The combination of any two of the following pathologies -- leucopenia, neutropenia, lymphocytopenia, decreased eosinophil count or monocytosis -- was taken as COVID-19-suggestive blood count changes. ${ }^{6,7}$

The following reference values were used for the diagnosis of the aforementioned changes. ${ }^{8}$ This crosssectional study was conducted at the antenatal care clinic of a tertiary University hospital between March and June 2020. All participants gave informed written consent before inclusion in the study. The study included 187 healthy pregnant females between the age of 18 and 40 years, irrespective of gestational age, who presented for routine antenatal care.

After informed consent had been obtained, all the study participants underwent detailed history taking, and general, chest, abdominal and local examinations. The aim of the detailed history and examinations was to assure the absence of any obstetric complications or chronic medical conditions. An emphasis was made during history taking and examinations to exclude any participant who were symptomatic for COVID-19 infection. The presence of any of the following: fever, dry cough, tiredness, muscle aches and pains, sore throat, diarrhea, conjunctivitis, headache, loss of taste or smell, skin rash, discoloration of fingers or toes, difficulty in breathing or shortness of breath, chest pain or deteriorated general condition, in the previous 14 days preceding enrollment in the study was taken as suggestive of COVID-19, and the participant was excluded from the study.

After assuring proper inclusion of participants as previously detailed, a venous blood sample ( $3 \mathrm{~mL}$, to $\mathrm{K}^{+}$EDTA containing tube) was taken from each of the participants for complete and 
differential blood counts, using an automated cell counter as well as stained peripheral blood smear microscopy, with emphasis on differential leucocyte count. The combination of any two of the following pathologies -- leucopenia, neutropenia, lymphocytopenia, decreased eosinophil count or monocytosis -- was taken as COVID-19-suggestive blood count changes. ${ }^{6,7}$

The following reference values were used for the diagnosis of the aforementioned changes. ${ }^{8}$ Table 1

Table 1. Polymerase Chain Reaction (PCR) for detection of SARS-CoV-2

\begin{tabular}{|l|c|}
\hline Type of cells & Absolute values \\
\hline Total leucocytes & 4 to $11 \times 10^{9} / \mathrm{L}$ \\
\hline Segmented neutrophils & 2.4 to $7.5 \times 10^{9} / \mathrm{L}$ \\
\hline Lymphocytes & 1 to $4.75 \times 10^{9} / \mathrm{L}$ \\
\hline Eosinophils & 0.03 to $0.45 \times 10^{9} / \mathrm{L}$ \\
\hline Monocytes & 0.1 to $0.7 \times 10^{9} / \mathrm{L}$ \\
\hline
\end{tabular}

Participants who showed $\mathrm{CBC}$ changes suggestive of COVID-19 were scheduled for real- time PCR testing for detection of SARS-CoV-2 antigens. The test was done by obtaining a nasopharyngeal swab. Nucleic acid extraction from collected samples followed by RT-PCR assay using TaqMan One-Step RT-PCR protocol was performed.

\section{Follow up}

All the participants who showed COVID19-suggestive CBC changes, whether they were PCR positive or negative, were followed for 14 days for the presence of any symptoms suggestive of COVID-19 infection. Those who were PCR positive were isolated at home and given an appropriate treatment protocol for asymptomatic COVID-19 confirmed cases while those who were PCR negative were isolated at home and given health education about proper sanitation, diet, exercise and fluid intake.

\section{Results}

The study included 187 women with gestational ages ranging between 14 and 36 weeks. The mean age of participants was 26.8 years, and none of them had any medical disorders.

Indeed, $5.3 \% \quad(n=10)$ of the study population showed $\mathrm{CBC}$ changes that were suggestive of COVID-19. When these patients were scheduled for nasopharyngeal swab as a PCR confirmatory test, $30 \%(n=3)$ of them were PCR positive (which represented $1.6 \%$ of the whole study population). Table 2

Table 2. Frequency and percentage of participants who showed COVID-19suggestive CBC changes and PCR confirmed cases in relation to the overall study population

\begin{tabular}{l|c|c|} 
& $\mathbf{N}(\mathbf{1 8 7})$ & percentage \\
\hline Normal CBC & 177 & $94.7 \%$ \\
\hline $\begin{array}{l}\text { COVID-19 suggestive } \\
\text { changes }\end{array}$ & 10 & $5.3 \%$ \\
\hline $\begin{array}{l}\text { PCR confirmed cases } \\
\text { n }\end{array}$ & 3 & $1.6 \%$ \\
\hline
\end{tabular}


The mean total leucocyte count among those ten cases that showed COVID-19suggestive changes was $3.7 \pm 0.26 \times 109 / L(3.2-4)$, while the mean neutrophil count was $2.5 \pm 0.26 \times 109 / L(2.3-2.9)$. The mean lymphocytes count was $0.98 \pm 0.13 \times 109 / L(0.8-1.2)$, while the mean eosinophil count was $0.08 \pm 0.06 \times 109 / L(0.02-0.15)$. Table 3

Table 3. Characteristics of peripheral blood leukocyte differential counts in participants who showed COVID-19suggestive changes

\begin{tabular}{|c|c|c|}
\hline & Mean $\pm S D$ & $\begin{array}{c}\text { Range } \\
\text { (min-max) }\end{array}$ \\
\hline $\begin{array}{c}\text { Total Leucocytic count } \\
\left(X 10^{9} / L\right)\end{array}$ & $3.7 \pm 0.26$ & $(3.2-4)$ \\
\hline $\begin{array}{c}\text { Lymphocyte count } \\
\left(X 10^{9} / L\right)\end{array}$ & $0.98 \pm 0.13$ & $(0.8-1.2)$ \\
\hline $\begin{array}{l}\text { Neutrophil count } \\
\qquad\left(X 10^{9} / L\right)\end{array}$ & $2.5 \pm 0.26$ & (2.3-2.9) \\
\hline $\begin{array}{c}\text { Eosinophil count } \\
\qquad\left(X 10^{9} / L\right)\end{array}$ & $0.08 \pm 0.06$ & $(0.02-0.15)$ \\
\hline $\begin{array}{r}\text { Monocyte count } \\
\left(X 10^{9} / \mathrm{L}\right)\end{array}$ & $0.26 \pm 0.14$ & $(0.2-0.6)$ \\
\hline
\end{tabular}

The most frequently encountered COVID-19-suggestive change in peripheral blood leucocyte differential counts was leucopenia $(100 \%$ of participants with $\mathrm{CBC}$ changes), followed by decreased eosinophil count $(50 \%)$, then neutropenia and lymphocytopenia (each represented in $30 \%$ of participants with $\mathrm{CBC}$ changes). Table 4
Table 4. Frequency and percentage of differential leucocyte count changes among participants who showed COVID-19-suggestive CBC changes

\begin{tabular}{|l|c|c|}
\hline & $\begin{array}{c}\text { Frequency } \\
(\mathrm{n}=10)\end{array}$ & Percentage \\
\hline Leucopenia & 10 & $100 \%$ \\
\hline Lymphopenia & 3 & $30 \%$ \\
\hline Neutropenia & 3 & $30 \%$ \\
\hline $\begin{array}{l}\text { Decreased Eosinophil } \\
\text { count }\end{array}$ & 5 & $50 \%$ \\
\hline $\begin{array}{l}\text { Increased Monocyte } \\
\text { count }\end{array}$ & 0 & $0 \%$ \\
\hline
\end{tabular}

When the 10 participants who showed COVID-19-suggestive differential blood count changes were followed for COVID-19 symptoms, three of them developed such symptoms (representing 30\%). Two of the three were PCR positive, which meant that $66.6 \%$ of the PCR positive asymptomatic pregnant participants developed symptoms later. On the other hand, one of the three participants who developed COVID-19 symptoms later tested PCR negative. Table 5

\section{Discussion}

Ever since the WHO has declared COVID-19 outbreaks to be pandemic, research worldwide has been mainly focused on the diagnosis and treatment of this disease. ${ }^{3}$ Initial reports came from Wuhan, Hubei Province of China, where the COVID-19 started. Subsequently, with the vast spread of the infection, reports of its spread came 
Proceedings in Obstetrics and Gynecology, 2021;10(2):5

from all over the world. From these reports it was evident that COVID-19 represents a wide panel of presentations, ranging from asymptomatic cases to severe, lifethreatening conditions. ${ }^{5}$

Table 5. Frequency and percentage of appearance of COVID-19 symptoms during the follow up period

Frequency of positive appearance of Percentage symptom during follow up

Suggestive CBC with positive PCR $(n=3)$

Suggestive $C B C$ with negative PCR $(n=7)$

Total

\begin{tabular}{|c|c|}
\hline 2 & $20 \%$ \\
\hline 1 & $10 \%$ \\
\hline $3(n=10)$ & $30 \%$ \\
\hline
\end{tabular}

\section{Discussion}

Ever since the $\mathrm{WHO}$ has declared COVID-19 outbreaks to be pandemic, research worldwide has been mainly focused on the diagnosis and treatment of this disease. ${ }^{3}$ Initial reports came from Wuhan, Hubei Province of China, where the COVID-19 started. Subsequently, with the vast spread of the infection, reports of its spread came from all over the world. From these reports it was evident that COVID-19 represents a wide panel of presentations, ranging from asymptomatic cases to severe, lifethreatening conditions. ${ }^{5}$

Several differential leucocyte count changes have been identified in conjunction with the diagnosis of COVID-19, including leucopenia, neutropenia, lymphocytopenia, decreased eosinophil count and monocytosis. However, the previous changes were not constant throughout patients, who presented with variable combinations of the abovementioned changes. $^{6,7}$

Khartabil et al., 2020 performed a systematic review representing a summary of the diagnostic and prognostic value of hemocytometry markers in COVID-19 patients. This summary included analysis of 250 articles that were published between December 2019 and May 2020. The authors concluded that leucopenia, lymphcytopenia and an elevated neutrophil/lymphocyte ratio are the most consistent abnormal hemocytometric findings in patients with COVID-19, and that these alterations may be present at any time during the course of the disease, even in confirmed asymptomatic cases, with a tendency to marked alteration in severe cases. ${ }^{9}$

Such a view that even asymptomatic patients may show CBC changes was confirmed by Yan Ma et al., 2020. They concluded that leucopenia was present in $27.1 \%$ of confirmed asymptomatic COVID-19 patients and that neutropenia was present in $9.1 \%$ of confirmed 
asymptomatic COVID-19 patients. ${ }^{10}$

Thus, these previous reports confirmed that certain changes in differential leucocyte count are a constant feature in COVID-19 patients, and that such changes are present even in confirmed asymptomatic patients. CBC is an essential investigation in routine antenatal care and pregnant females are considered a higher risk group for COVID-19. In this study, we interpreted early detection of the presence of $\mathrm{CBC}$ changes as suggesting COVID-19 infection among asymptomatic pregnant females coming for routine antenatal care. With this goal in mind, we correlated these COVID-19 suggestive changes with the presence or absence of confirmed disease.

In our study, ten women showed $\mathrm{CBC}$ changes that are suggestive of COVID19 , and were scheduled for a nasopharyngeal swab for a PCR confirmatory test. Of these 10 women, $30 \%(n=3)$ of them were PCR positive (which represented $1.6 \%$ of the whole study population). This finding suggests the importance of leucocyte differential count results as support for early diagnosis of COVID-19 among asymptomatic pregnant females during this pandemic era, as $30 \%$ of asymptomatic pregnant females with CBC findings suggestive of COVID-19 changes were found to have confirmed disease (were PCR positive).

The mean total leucocyte count among those ten cases that showed COVID-19 suggestive changes denoted mild leucopenia. Of note, the CBC suggestive COVID-19 changes that we found in this research are compatible with Li et al., where they concluded that COVID-19 patients showed relatively lower absolute white blood cell (WBC) count $4.95(3.90,6.03) \times 10(9) / \mathrm{L}$, lymphocyte absolute count $1.20(0.98,1.50) \times 10(9) / L$ and eosinophil absolute count $0.01(0.01,0.01) \times 10(9) / L{ }^{6}$

In the present study, the most frequently encountered COVID-19 suggestive change in peripheral blood leucocyte differential counts was leucopenia $(100 \%)$, followed by decreased eosinophil count (50\%), then neutropenia and lymphocytopenia $(30 \%)$. In the Fan et al., study, leucopenia was observed in $29.2 \%$ of patients, while lymphocytopenia featured in $36.9 \%$ of patients. ${ }^{7}$ Li et al., noted that leucopenia was observed in $20 \%$ of patients, lymphocytopenia was featured in $20 \%$ of patients, while decreased eosinophil count was noted in $70 \%$ of cases. ${ }^{6}$

The differences between the findings in differential leukocyte count in this study and other studies came from the fact that other studies concluded their results from the study of CBC of COVID-19 confirmed patients, while our study started with the investigation of COVID19 suggestive CBC changes prior to confirmation of infection. When the ten participants who showed COVID-19 suggestive differential leucocyte count changes were followed for COVID-19 symptoms, three of them developed such symptoms (representing 30\%). Notably, two of the three were PCR positive, which meant that $66.6 \%$ of the 
PCR positive asymptomatic pregnant participants developed symptoms at a later phase of the infection. On the other hand, the one of the three participants who developed COVID-19 symptoms later was PCR negative, and when the PCR was repeated after appearance of symptoms, it was found to be positive, indicating that $\mathrm{CBC}$ changes suggestive of COVID-19 may even have a role in diagnosis of PCR negative cases.

This idea is consistent with Zhou et al., where they followed 13 asymptomatic confirmed COVID-19 cases for two weeks. They noted the presence of leucopenia in $15.4 \%$ of cases while $23.1 \%$ developed symptoms within two days following admission. ${ }^{11}$

Given that the findings of this study are so small $(n=3)$ as to be more anecdotal than statistically significant, they still pose some interesting questions for future research. In fact, $100 \%$ of patients that showed leucopenia, decreased eosinophil count, neutropenia or lymphocytopenia eventually tested positive for COVID-19 using PCR. If these findings can be replicated in a substantially larger population, they may show that COVID19-suggestive changes in differential blood counts may allow physicians identify asymptomatic COVID-19 patients, especially those with nascent disease or those who have verified exposures to the disease, more rapidly than is possible with existing PCR testing.

\section{Limitations of the study}

One of the main limitations of the study was the small number of women included. Furthermore, it would have been better if we had performed PCR testing for the entire study population as it would have given us a precise estimation of the prevalence of COVID19 among asymptomatic pregnant females coming for routine antenatal care. However, the regional protocol at the time of this study offered PCR testing only for symptomatic patients. In addition, replication of this study with a larger population would have further confirmed the role of changes in differential blood counts as predictors of nascent COVID-19.

\section{Conclusions}

Certain differential leucocyte count changes (leucopenia, neutropenia, lymphocytopenia and decreased eosinophil count) among asymptomatic pregnant women might be related to COVID-19 infection and, as such, they may be useful predictors of the need for further testing.

\section{References}

1. 2019 Novel coronavirus, Wuhan, China. Information for Healthcare Professionals. Centers for Disease Control and Prevention. https://www.cdc.gov/coronavirus/2019nCoV/hcp/index.html (Accessed on July 14, 2020). 
2. Jeffrey $\mathrm{K}$ Aronson. Coronaviruses $-\mathrm{a}$ general introduction Centre for Evidence-Based Medicine, Nuffield Department of Primary Care Health Sciences, University of Oxford. March 25, 2020. https://www.cebm.net/covid19/coronaviruses-a-general-introduction/

3. Director-General's remarks at the media briefing on 2019-nCoV on 12 July 2020. World Health Organization. https://www.who.int/dg/speeches/detail/ who-director-general-s-remarks-at-themedia-briefing-on-2019-ncov-on-11february-2020 (Accessed on July 12, 2020).

4. Zhou $P$, Yang $X L$, Wang $X G$, Hu $B$, Zhang L, Zhang W, Si HR, Zhu Y, Li B, Huang $\mathrm{CL}$, Chen HD, Chen J, Luo $Y$, Guo $H$, Jiang RD, Liu MQ, Chen $Y$, Shen XR, Wang $X$, Zheng XS, Zhao $K$, Chen QJ, Deng F, Liu LL, Yan B, Zhan FX, Wang YY, Xiao GF, Shi ZL. A pneumonia outbreak associated with a new coronavirus of probable bat origin. Nature. 2020 Mar;579(7798):270-273. https://doi.org/10.1038/s41586-020-

2012-7. Epub 2020 Feb 3. PMID: 32015507 ; PMCID: PMC7095418.

5. Wu Z, McGoogan JM. Characteristics of and Important Lessons From the Coronavirus Disease 2019 (COVID-19) Outbreak in China: Summary of a Report of 72314 Cases From the Chinese Center for Disease Control and Prevention. JAMA. 2020 Apr 7;323(13):1239-1242. https://doi.org/10.1001/jama.2020.2648. PMID: 32091533.

6. Li YX, Wu W, Yang T, Zhou W, Fu YM, Feng QM, Ye JM. [Characteristics of peripheral blood leukocyte differential counts in patients with COVID-19]. Zhonghua Nei Ke Za Zhi. 2020 Mar 1;59(0):E003. Chinese. Epub ahead of print. PMID: 32114745.
7. Fan BE, Chong VCL, Chan SSW, Lim $\mathrm{GH}$, Lim KGE, Tan GB, Mucheli SS, Kuperan $\mathrm{P}$, Ong $\mathrm{KH}$. Hematologic parameters in patients with COVID-19 infection. Am J Hematol. 2020 Jun;95(6):E131-E134. https://doi.org/10.1002/ajh.25774. Epub 2020 Mar 19. Erratum in: Am J Hematol. 2020 Nov;95(11):1442. PMID: 32129508 .

8. Choladda Vejabhuti Curry. Differential Blood Count, Medscape, Updated: Nov 20, 2019.

9. Khartabil TA, Russcher $\mathrm{H}$, van der Ven $A$, de Rijke YB. A summary of the diagnostic and prognostic value of hemocytometry markers in COVID-19 patients. Crit Rev Clin Lab Sci. 2020 Sep;57(6):415-431. https://doi.org/10.1080/10408363.2020. 1774736. Epub 2020 Jun 22. PMID: 32568604 .

10. Ma $Y, X u$ QN, Wang FL, Ma XM, Wang $X Y$, Zhang $X G$, Zhang ZF. Characteristics of asymptomatic patients with SARS-CoV-2 infection in Jinan, China. Microbes Infect. 2020 MayJun;22(4-5):212-217.

https://doi.org/10.1016/j.micinf.2020.04. 011. Epub 2020 May 7. PMID: 32387682; PMCID: PMC7204664.

11. Zhou X, Li Y, Li T, Zhang W. Follow-up of asymptomatic patients with SARSCoV-2 infection. Clin Microbiol Infect. $2020 \quad J u l ; 26(7): 957-959$. https://doi.org/10.1016/i.cmi.2020.03.02 4. Epub 2020 Mar 28. PMID: 32234453; PMCID: PMC7271011. 